\title{
Role of circulating S100A4 protein in obesity: a case-control study in prepuberal children
}

\author{
Andrea Méndez-Gutierrez ${ }^{1}$, Augusto Anguita-Ruiz ${ }^{1,2}$, Azahara I. Ruperez ${ }^{3,2}$, Rosaura Leis ${ }^{4,2}$, \\ Gloria Bueno $^{3,2}$, Mercedes Gil-Campos, ${ }^{5,2}$, ANGEL. GIL ${ }^{1,2}$ and Concepción M. Aguilera ${ }^{1,2}$ \\ ${ }^{1}$ Department of Biochemistry and Molecular Biology II, Institute of Nutrition and Food Technology, Biomedical \\ Reasearch Center, University of Granada, Granada, Spain, \\ ${ }^{2}$ CIBER Fisiopatología de la Obesidad y la Nutrición (CIBEROBN), Madrid, Spain, \\ ${ }^{3}$ Growth, Exercise, Nutrition and Development (GENUD) Research Group, University of Zaragoza, Zaragoza, Spain., \\ ${ }^{4}$ Unit of Investigation in Nutrition, Growth and Human Development of Galicia, Pediatric Department, University of \\ Santiago de Compostela, Santiago de Compostela, Spain and \\ ${ }^{5}$ Department of Paediatrics, Reina Sofia University Hospital, Institute Maimónides of Biomedicine Investigation of \\ Córdoba (IMIBIC), University of Córdoba, Córdoba, Spain
}

\begin{abstract}
Introduction: Childhood obesity is considered one of the most serious public health problems of the 21st century. Obesity-associated inflammation could be one of the mechanisms that triggers insulin resistance that could drive systemic alterations such as metabolic disorder. Recently, circulating levels of S100A4 has been associated with insulin resistance and subcutaneuous white adipose tissue inflammation independently of body mass index (BMI) in a cohort of obese adults. Nonetheless, the link between S100A4 and insulin resistance in children is still not known yet. Thus, the aim of the study was to determine if S100A4 plasma levels were associated with insulin resistance status in a cohort of prepuberal children.
\end{abstract}

Material and methods: In this case-control multicentre study, 250 prepuberal children took part and were stratified in six groups according to sex, obesity stage and insulin resistance status. Blood samples were withdrawn in resting conditions after an overnight fasting. Anthropometric measurements and a routine biochemical analyses were performed. Homeostasis model assessment for insulin resistance index (HOMA-IR) was calculated using fasting plasma glucose and insulin values. S100A4 plasma levels were determined by ELISA CSBEL02032HU (Cusabio Biotech, Wuhan, China).

Results: A lineal multiple regresión $(\alpha=0.05)$ identified a significative association between S100A4 plasma levels and HOMA-IR in the cohort; each HOMA-IR increasing unit correlated with an increase of $0.008 \mathrm{mg} / \mathrm{dL}$ in S100A4 plasma levels. (SE $=0.003$ and $p=0.02$ ). Moreover, we also observed a positive significative association between S100A4 plasma levels and glucose blood levels $(p=0.005)$ and BMI $(p=0.008)$. Inter-group comparations analyses revealed significative differences between normal-weight and insulino-resistant obese boys $(p=0.024)$. The same result was obtained between normal-weight and insulino-resistant obese girls $(p=0.04)$, finding a higher S100A4 concentration in insulino- resistant children. As expected, plasma S100A4 levels were also higher in obese children versus normal-weight children $(p=0.02)$.

Discussion: These data could be clinical relevant due to the possible potential of S100A4 protein as a new circulating biomarker of resistance insulin in a cohort of prepuberal children. These results are supported by other studies in obese adults and adolescents. In conclusion, these results suggest that S100A4 is associated with obesity and insulin resistance in prepuberal children. However, more studies are needed to study the implication and mechanism of this protein in the development of insulin resistance.

\section{Conflict of Interest}

There is no conflict of interest 